\title{
TU/e EmonOWEN

\section{Simulation of bubble growth during the foaming process and mechanics of the solid foam}

\section{Citation for published version (APA):}

Mitrias, C., Egelmeers, T. R. N., Jaensson, N. O., Hulsen, M. A., \& Anderson, P. D. (2019). Simulation of bubble growth during the foaming process and mechanics of the solid foam. Rheologica Acta, 58(3-4), 131-144.

https://doi.org/10.1007/s00397-018-01123-x

DOI:

10.1007/s00397-018-01123-x

Document status and date:

Published: 01/04/2019

\section{Document Version:}

Accepted manuscript including changes made at the peer-review stage

\section{Please check the document version of this publication:}

- A submitted manuscript is the version of the article upon submission and before peer-review. There can be important differences between the submitted version and the official published version of record. People interested in the research are advised to contact the author for the final version of the publication, or visit the $\mathrm{DOI}$ to the publisher's website.

- The final author version and the galley proof are versions of the publication after peer review.

- The final published version features the final layout of the paper including the volume, issue and page numbers.

Link to publication

\section{General rights}

Copyright and moral rights for the publications made accessible in the public portal are retained by the authors and/or other copyright owners and it is a condition of accessing publications that users recognise and abide by the legal requirements associated with these rights.

- Users may download and print one copy of any publication from the public portal for the purpose of private study or research.

- You may not further distribute the material or use it for any profit-making activity or commercial gain

- You may freely distribute the URL identifying the publication in the public portal.

If the publication is distributed under the terms of Article $25 \mathrm{fa}$ of the Dutch Copyright Act, indicated by the "Taverne" license above, please follow below link for the End User Agreement:

www.tue.nl/taverne

Take down policy

If you believe that this document breaches copyright please contact us at:

openaccess@tue.nl

providing details and we will investigate your claim. 


\title{
Simulation of bubble growth during the foaming process and mechanics of the solid foam
}

\author{
C. Mitrias ${ }^{1}$, T.R.N. Egelmeers ${ }^{1}$, N.O. Jaensson ${ }^{1}$, M.A. Hulsen ${ }^{1}$, \\ P.D. Anderson ${ }^{1}$
}

Department of Mechanical Engineering, Eindhoven University of Technology, P.O. Box 513, 5600 MB, Eindhoven, The Netherlands

Received: date / Revised version: date

\begin{abstract}
Elastomeric foams are widely used in different types of applications where different material properties are of interest in each application. All of these properties are governed by the microstructure and the properties of the material matrix. Studying the evolution of the microstructure experimentally is extremely challenging. Thus here, we use direct numerical simulations to gain an insight into the changes that happen from the creation of the gas bubbles in the liquid state, until the solidification into a cellular morphology. Furthermore, the resulting microstructure is then used directly in simulations of solid mechanical testing to determine the mechanical properties of the foam. The matrix fluid is assumed to be Newtonian and incompressible. A linear elastic isotropic material model for the solidified polymer was used to obtain the solid foam properties. The foam was described by a representative volume element (RVE), where a small number of bubbles was randomly distributed. Using this approach, the RVE can describe the bulk behaviour of the foam, while remaining computationally tractable. Microstructures with volumes fraction of over 90\% (2D) and 45\% (3D) are accurately captured. In addition, the influence that the bubble growth rate and the initial bubble distribution of the fluid simulations have on the solid foam properties was studied.
\end{abstract}

\section{Introduction}

There are several studies in the literature regarding the solid mechanics of foams. When open cell foams are considered, the microstructure of these

Send offprint requests to: 
foams can be modeled as clusters of Kelvin cells, where one Kelvin cell consists of beam and spring elements [1-4]. Using compression and shear simulations the mechanical properties and the energy absorption are obtained. However, these simulations are only accurate for foams in a small range of high-volume fractions [3]. Furthermore, during processing, microstructures deform and do not agree with Kelvin cells. Closed-cell foams are used more for engineering applications, since they are stronger and can absorb more energy. It is therefore interesting to investigate the solid mechanics of closed-cell foam. The mechanical properties of foams are usually assumed to only dependent on the solid properties and the relative density of the foam [5-7]. However, processing conditions, i.e. flow, can have a significant influence on the microstructures being formed, and therefore on the macroscopic properties of the foam.

Existing research on foams mostly focuses on either the solid mechanical properties of an existing foam or on the foaming process [8-15]. The objective of this article is to connect the foaming process with the solid mechanical properties. The solid mechanical properties are determined using the microstructures generated in the foaming process. As a result, in the deformation simulations standard solid elements are applied, which describe the microstructures of the foams in more detail, compared to beam and spring elements. Furthermore, using this approach, closed-cell foams with realistic microstructures spanning a large volume fraction range of bubbles in the bulk can be generated. To the best of our knowledge, this is the first time where solid mechanical properties are determined on microstructures generated by modeling the foaming process using direct numerical simulations.

In order to perform this analysis, first in Section 2, the models for the bubble growth during foaming and the solid mechanics will be described. In Section 3, results will be presented of the obtained microstructures both in 2D and 3D. Furthermore, the mechanical properties of this structures will be obtained using compression and shear simulations. Finally, in Section 4 we close with conclusions.

\section{Problem definition}

For the purpose of this work, a number of bubbles in a Newtonian liquid is randomly distributed in a periodic domain and an internal pressure is applied, such that the bubbles start to grow. When a certain volume fraction is reached, we assume that the material solidifies and the obtained microstructure is used in solid mechanics simulations. In this section, both of the problems for bubble growth and solid mechanics will be defined. The finite element method is used to find an approximate solution. Initially, we will derive a two-dimensional model which will be extended to three dimensions. 


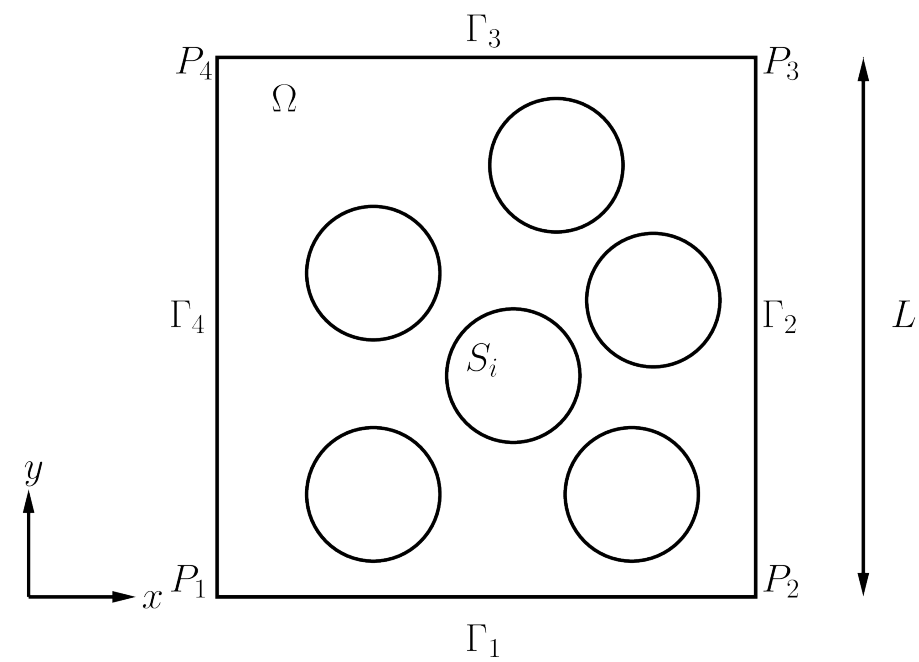

Fig. 1: Schematic representation of a square domain RVE $\Omega$, including the box boundaries, $\Gamma_{i}$, the points, $P_{i}$, with $i=1, \ldots, 4$, the bubble boundaries, $S_{i}$, with $i=1,2, \ldots, n_{\mathrm{b}}$ and length $L$.

\subsection{Bubble growth model}

The objective of the fluid simulation is to predict the growth of gas bubbles in a closed-cell foam and end up with a realistic microstructure. Initially, small circular bubbles are assumed to be present in a square Representative Volume Element (RVE). In Fig. 1, an example of a square RVE with side length $L$, containing randomly distributed monodisperse bubbles is shown. The domain $\Omega$ is visible with outer box boundaries $\Gamma=\cup_{i=1}^{4} \Gamma_{i}$, bubble interface boundaries $S=\cup_{i=1}^{n_{\mathrm{b}}} S_{i}$, where $n_{\mathrm{b}}$ the number of bubbles, and corner points $P_{i}$. For simplicity of the graphical representation the outer box boundaries are straight. In the simulation, the bubbles will grow until a certain volume fraction of bubbles in the bulk is reached. The volume fraction plays a large role in describing the mechanical behavior of foams $[5]$.

2.1.1 Governing equations Since we are mainly focused on highly-viscous polymeric liquids we assume inertial forces can be neglected. Furthermore, the foaming fluid is assumed to be incompressible and Newtonian. As a result, the momentum and the mass balance reduce to

$$
\begin{aligned}
& -\boldsymbol{\nabla} \cdot \boldsymbol{\sigma}=\mathbf{0} \quad \text { in } \Omega, \\
& \boldsymbol{\nabla} \cdot \boldsymbol{u}=0 \quad \text { in } \Omega \text {, }
\end{aligned}
$$

where $\boldsymbol{u}$ is the velocity vector and $\boldsymbol{\sigma}$ denotes the Cauchy stress tensor. For incompressible Newtonian fluids the stress tensor $\boldsymbol{\sigma}$ is written as:

$$
\boldsymbol{\sigma}=-p \boldsymbol{I}+2 \mu \boldsymbol{D}
$$


where $p$ is the pressure, $\boldsymbol{I}$ is the identity tensor, $\mu$ is the viscosity of the matrix fluid and $\boldsymbol{D}=\left(\boldsymbol{\nabla} \boldsymbol{u}+(\boldsymbol{\nabla} \boldsymbol{u})^{T}\right) / 2$ is the symmetric part of the velocity gradient tensor.

2.1.2 Boundary and interface conditions The balance equations are closed by imposing periodic boundary conditions for the velocity and by imposing a hydrostatic pressure on the inside of the bubble interface, to make the bubbles grow. The rate at which the domain grows cannot be estimated a-priori, so the velocity of the outer boundaries are unknowns. We assume that the outer boundaries are force-free, leading to

$$
\begin{aligned}
& \left.\boldsymbol{u}\right|_{\Gamma_{2}}=\left.\boldsymbol{u}\right|_{\Gamma_{4}}+U e_{x}, \\
& \left.\boldsymbol{u}\right|_{\Gamma_{3}}=\left.\boldsymbol{u}\right|_{\Gamma_{1}}+V e_{y} .
\end{aligned}
$$

The unknown velocity components $U, V$ are such, that the force free constraints

$$
\begin{aligned}
& \int_{\Gamma_{2,4}} t_{x} d \Gamma=0, \\
& \int_{\Gamma_{1,3}} t_{y} d \Gamma=0,
\end{aligned}
$$

are fulfilled. Herein, $\boldsymbol{t}=\boldsymbol{\sigma} \cdot \boldsymbol{m}$ is the traction vector $\boldsymbol{m}$ the outwardly directed unit-normal vector on the outer box boundaries $\Gamma$. The traction acting on the liquid-gas interface boundaries, $S_{i}$, has two contributions: the hydrostatic pressure and the surface tension, and is expressed as follows

$$
\left.\boldsymbol{t}\right|_{S_{i}}=-p_{\mathrm{b}} \boldsymbol{n}+\gamma \kappa \boldsymbol{n},
$$

where $\gamma$ is the surface tension, $\kappa$ the curvature, and $\boldsymbol{n}$ is the outwardly directed unit-normal vector on the bubble boundaries $S_{i}$. To ensure growth of the bubbles, the hydrostatic pressure, $p_{\mathrm{b}}$ should be a fraction higher than the estimated Laplace pressure generated by surface tension. The hydrostatic pressure is calculated at each time step and for each individual bubble. This is done by calculating the area occupied by each bubble and obtaining the radius of a circular bubble with equivalent area. In reality this process is of course more complicated and the dynamics do not depend only on the hydrostatic pressure. For that the diffusion of the gas into the bubbles should be taken into account, a process that several authors have studied in the literature $[9,16,17]$. In $2 \mathrm{D}$ the Laplace pressure can be estimated by $\Delta p_{\mathrm{La}}=\gamma / R$, with $R$ the mean radius of the bubble, where $\gamma$ is taken constant. The hydrostatic pressure is given by $p_{\mathrm{b}}=\alpha_{\mathrm{b}} \Delta p_{\text {La }}$ where $\alpha_{\mathrm{b}}$ is defined as the growth parameter. The growth parameter influences the geometry of the microstructure. 
2.1.3 Interface tracking The sharp interface between the bubbles and the matrix fluid, $S_{i}$, is described by a line. The interface is tracked by following the motion of this line, the velocity of the interface $\dot{\boldsymbol{x}}$ must be such that:

$$
\dot{\boldsymbol{x}} \cdot \boldsymbol{n}=\boldsymbol{u} \cdot \boldsymbol{n} \quad \text { on } S .
$$

After manipulation of Eq. (9), the velocity of the interface is implemented in normal direction $[18,19]$

$$
\dot{\boldsymbol{x}}+\left(\boldsymbol{u}-\boldsymbol{u}_{c}\right) \cdot \nabla_{s} \boldsymbol{x}=\boldsymbol{u} \quad \text { on } S,
$$

where $\boldsymbol{u}_{c}$ is the velocity of the center of volume of the bubble, $\boldsymbol{u}$ the material velocity at the interface and $\nabla_{s}$ is the surface gradient operator.

2.1.4 Weak form The weak form is obtained by multiplying Eqs. (1), (2) and (10) with test functions and after applying partial integration and Gauss' theorem, we obtain the following weak form: Find $\boldsymbol{u}, p, \boldsymbol{x}$ such that:

$$
\begin{aligned}
\left((\boldsymbol{\nabla} \boldsymbol{v})^{\mathrm{T}}, 2 \mu \boldsymbol{D}\right)-(\boldsymbol{\nabla} \cdot \boldsymbol{v}, p) & =\left\langle\boldsymbol{v}, p_{\mathrm{b}} \boldsymbol{n}\right\rangle_{S}-\langle\boldsymbol{v}, \gamma \kappa \boldsymbol{n}\rangle_{S} \\
& \text { for all } \boldsymbol{v}, \\
(q, \boldsymbol{\nabla} \cdot \boldsymbol{u}) & =0 \\
& \text { for all } q, \\
\langle\boldsymbol{w}, \dot{\boldsymbol{x}}\rangle_{S}+\left\langle\boldsymbol{w},\left(\boldsymbol{u}-\boldsymbol{u}_{\mathrm{c}}\right) \cdot \nabla_{s} \boldsymbol{x}\right\rangle_{S} & =\langle\boldsymbol{w}, \boldsymbol{u}\rangle_{S} \\
& \text { for all } \boldsymbol{w},
\end{aligned}
$$

where $(a, b)$ is the standard $L_{2}$ inner product on $\Omega$ and $\langle a, b\rangle_{S}$ on surface $S$. The boundary conditions in Eqs. (4) and (5) are implemented using Lagrangian multipliers.

2.1.5 Discretization The finite element method is used to find an approximate solution. Taylor-Hood elements are employed, hence quadratic interpolation for the velocity and linear interpolation for the pressure is used. The surface mesh on $S_{i}$ consists of quadratic line elements. A second-order time discretization (BDF2) is used for the interface tracking with

$$
\dot{\boldsymbol{x}}=\frac{3 \boldsymbol{x}^{n+1}-4 \boldsymbol{x}^{n}+\boldsymbol{x}^{n-1}}{2 \Delta t},
$$

where $\Delta t$ is the time step. Solving a decoupled system of equations, the time step is limited by the mesh capillary time which is $\Delta t=\Delta x \mu / \gamma$, where $\Delta x$ is the element size [20]. Eqs. (11)-(13) are solved in a coupled approach to make the time step independent of the characteristic mesh size time limit. The coupled equations form a non-linear system of equations which is linearized using a Newton-like iterative scheme where

$$
\begin{aligned}
& \boldsymbol{u}=\boldsymbol{u}_{i}+\delta \boldsymbol{u}, \\
& \boldsymbol{x}=\boldsymbol{x}_{i}+\delta \boldsymbol{x},
\end{aligned}
$$


for the velocity and position respectively. Subscript $i$ indicates the current iteration, $\delta \boldsymbol{u}$ is the difference in velocity after one iteration and $\delta \boldsymbol{x}$ is the displacement of the nodes after one iteration. In the coupled equations, the position $\boldsymbol{x}$ as an unknown only exists on the bubble interface, therefore the iterative scheme is not fully Newton-Raphson. The surface tension term is linearized and rewritten in terms of curvilinear coordinates, as follows:

$$
\begin{gathered}
\langle\boldsymbol{v}, \gamma \kappa \boldsymbol{n}\rangle_{S}=\gamma\left\langle\frac{\partial \boldsymbol{v}}{\partial \xi^{\alpha}}, \boldsymbol{g}^{\alpha}\right\rangle_{S}- \\
\gamma\left\langle\frac{\partial \boldsymbol{v}}{\partial \xi^{\alpha}} \cdot \boldsymbol{g}^{\beta}, \frac{\partial \boldsymbol{v}}{\partial \xi^{\alpha}} \cdot \boldsymbol{g}^{\beta}\right\rangle_{S}+ \\
\gamma\left\langle\frac{\partial \boldsymbol{v}}{\partial \xi^{\alpha}} \cdot \boldsymbol{g}^{\alpha}, \frac{\partial \boldsymbol{v}}{\partial \xi^{\beta}} \cdot \boldsymbol{g}^{\beta}\right\rangle_{S}
\end{gathered}
$$

where $\boldsymbol{g}^{\alpha}, \boldsymbol{g}_{\alpha}$ the covariant and contravariant base vectors. The use of a curvilinear coordinate system allows the coordinate lines to be curved. The change in hydrostatic pressure, due to the volume changes, is accounted for every iteration. Eq. (13) is rewritten in curvilinear coordinates and linearized, as follows

$$
\begin{aligned}
& \left\langle\boldsymbol{w}, \frac{3 \boldsymbol{x}-4 \boldsymbol{x}^{n}+\boldsymbol{x}^{n-1}}{2 \Delta t}\right\rangle_{S}+\left\langle\boldsymbol{w}, \frac{3 \delta \boldsymbol{x}}{2 \Delta t}\right\rangle_{S} \\
+ & \left\langle\boldsymbol{w}, \frac{3 \boldsymbol{x}-4 \boldsymbol{x}^{n}+\boldsymbol{x}^{n-1}}{2 \Delta t} \boldsymbol{g}^{\alpha} \cdot \frac{\partial \delta \boldsymbol{x}}{\partial \xi^{\alpha}}\right\rangle_{S} \\
+ & \left\langle\boldsymbol{w},\left(\boldsymbol{u}-\boldsymbol{u}_{c}\right) \cdot \boldsymbol{g}^{\alpha} \frac{\partial \boldsymbol{x}}{\partial \xi^{\alpha}}\right\rangle_{S}-\langle\boldsymbol{w}, \boldsymbol{u}\rangle_{S} \\
+ & \left\langle\boldsymbol{w}, \delta \boldsymbol{u} \cdot \boldsymbol{g}^{\alpha} \frac{\partial \boldsymbol{x}}{\partial \xi^{\alpha}}\right\rangle_{S}-\langle\boldsymbol{w}, \delta \boldsymbol{u}\rangle_{S} \\
- & \left\langle\boldsymbol{w},\left(\boldsymbol{u}-\boldsymbol{u}_{c}\right) \cdot \boldsymbol{g}^{\alpha} \cdot \frac{\partial \delta \boldsymbol{x}}{\partial \xi^{\beta}} \boldsymbol{g}^{\beta} \frac{\partial \boldsymbol{x}}{\partial \xi^{\alpha}}\right\rangle_{S} \\
+ & \left\langle\boldsymbol{w},\left(\boldsymbol{u}-\boldsymbol{u}_{c}\right) \cdot \boldsymbol{g}^{\alpha} \frac{\partial \delta \boldsymbol{x}}{\partial \xi^{\alpha}}\right\rangle_{S} \\
+ & \left\langle\boldsymbol{w},\left(\boldsymbol{u}-\boldsymbol{u}_{c}\right) \cdot \boldsymbol{g}^{\alpha} \frac{\partial \boldsymbol{x}}{\partial \xi^{\alpha}} \boldsymbol{g}^{\beta} \cdot \frac{\partial \delta \boldsymbol{x}}{\partial \xi^{\beta}}\right\rangle_{S} \\
- & \left\langle\boldsymbol{w}, \boldsymbol{u} \boldsymbol{g}^{\alpha} \cdot \frac{\partial \delta \boldsymbol{x}}{\partial \xi^{\alpha}}\right\rangle_{S}=0 .
\end{aligned}
$$

Eqs. (15)-(18) are substituted in Eqs. (11)-(13) to obtain the final weak form. The system of equation can be solved by determining the unknowns $\delta \boldsymbol{u}, \delta \boldsymbol{x}$ and $p$. The converged solution is obtained when the maximum difference of $\boldsymbol{r}_{x, i}=\left|\boldsymbol{x}_{i}-\boldsymbol{x}_{i-1}\right|, \boldsymbol{r}_{u, i}=\left|\boldsymbol{u}_{i}-\boldsymbol{u}_{i-1}\right|$ and $r_{p, i}=\left|p_{i}-p_{i-1}\right|$ of two successive iterations is less than $r_{x, \max }<\epsilon|| b||_{2}, r_{u, \max }<\epsilon\|b\|_{2}$ and $r_{p, \max }<\epsilon|| b \|_{2}$, where in this work $\epsilon=10^{-7}$ is fixed and $\|b\|_{2}$ is the $L^{2}$ norm of the right hand side. A direct solver from the HSL library [21] is used to solve the system of equations. 
2.1.6 Mesh movement At every iteration the nodes of the mesh are updated by solving a Poisson problem with the solution $\delta \boldsymbol{x}$ of the interface motion as boundary conditions

$$
\begin{aligned}
\boldsymbol{\nabla} \cdot(k \boldsymbol{\nabla}(\Delta \boldsymbol{d})) & =0 \quad \text { in } \Omega, \\
\Delta \boldsymbol{d} & =\left.\delta \boldsymbol{x}\right|_{S}, \\
\Delta \boldsymbol{d} & =\left.\delta \boldsymbol{x}\right|_{\Gamma},
\end{aligned}
$$

where $k$ is a constant diffusion coefficient and $\Delta \boldsymbol{d}$ are the nodal mesh displacements $[18,22]$. The movement of the outer boundaries, denoted by $\left.\delta \boldsymbol{x}\right|_{\Gamma_{i}}$ is, unlike $\left.\delta \boldsymbol{x}\right|_{S_{i}}$, not known after solving the system of equations. It is solved by a first-order integration of the velocity over the time: $\left.\delta \dot{\boldsymbol{x}}\right|_{\Gamma_{i}}=\left.\delta \boldsymbol{u}\right|_{\Gamma_{i}}$.

The mesh coordinates in the new iteration are obtained using the nodal mesh displacements, as follows

$$
\boldsymbol{d}_{i+1}=\boldsymbol{d}_{i}+\Delta d .
$$

Every time step during the simulation the aspect ratio and the area is computed for all elements. The domain is remeshed if the change in area or the change in aspect ratio of one element is larger than a threshold [22]. Furthermore, mesh refinement is used to accurately describe the fluid dynamics between two bubble interfaces. A first-order time integration scheme is used when the mesh is either refined or remeshed. A line element on the bubble interface is refined if

$$
L_{\text {side }}>\frac{L_{\text {dist }}}{R_{\text {crit }}},
$$

where $R_{\text {crit }}$ is the refinement criteria, $L_{\text {dist }}$ the distance between the node in the center of the element to the closest node on a different curve and $L_{\text {side }}$ is the length of the element, see Fig. 2. If refinement of the line element is needed, the quadratic line element is split, creating one additional line element on the curve. For meshing the domain, we use Gmsh [23] which will automatically create smaller triangular elements connected to the additional node, resulting in more elements between the bubble interfaces.

2.1.7 Extension of bubble growth model in 3D The extension of the model to three dimensions is relatively straightforward. The corresponding schematic representation can be seen in Fig. 3. The addition of the constraints in the third direction is required

$$
\left.\boldsymbol{u}\right|_{\Gamma_{5}}=\left.\boldsymbol{u}\right|_{\Gamma_{6}}+W \boldsymbol{e}_{z},
$$

where the additional unknown velocity component $W$ is such, that the force free constraint in the $z$-direction

$$
\int_{\Gamma_{5,6}} t_{z} d \Gamma=0
$$




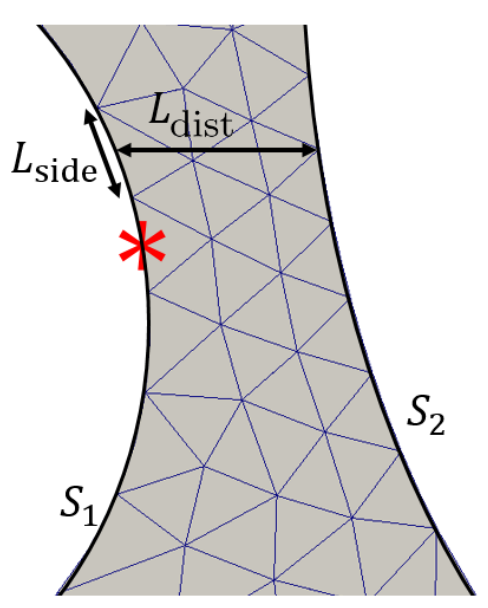

(a)

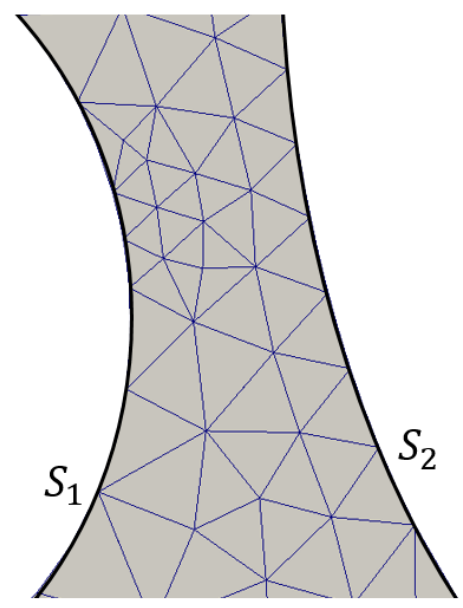

(b)

Fig. 2: a) The mesh before mesh refinement. One line element on curve $S_{1}$ is marked for refinement $(*)$. b) The mesh after mesh refinement, the marked line element is split in two, creating an additional line element on the bubble interface.

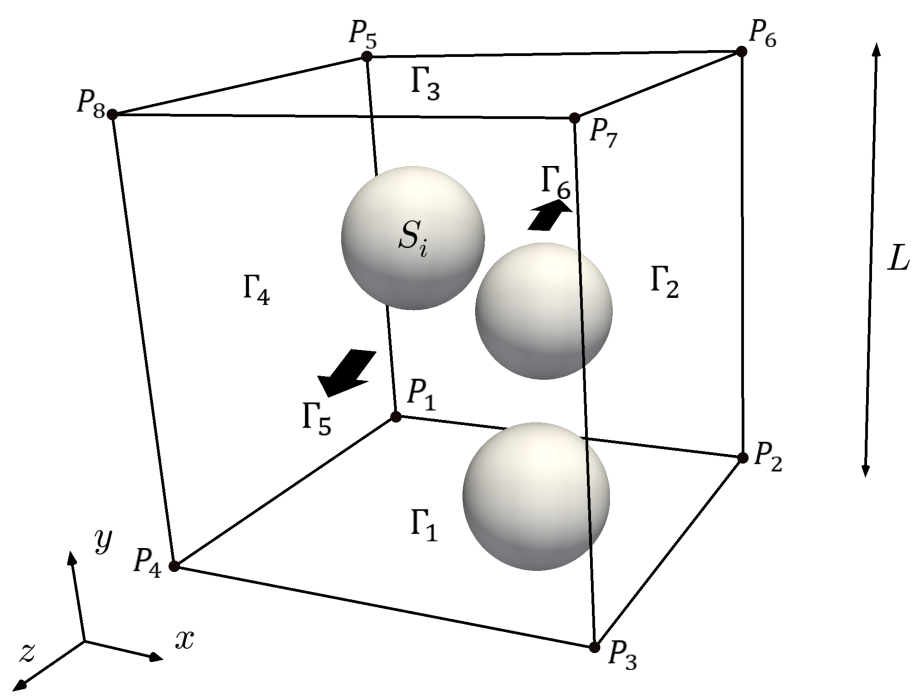

Fig. 3: Schematic representation of a cubic domain RVE $\Omega$, including the box boundaries, $\Gamma_{i}$, the points, $P_{i}$, with $i=1, \ldots, 6$ the bubble boundaries, $S_{i}$, with $i=1,2, \ldots, n_{\mathrm{b}}$ and length $L$. 
is fulfilled. However, the refinement of the mesh is more cumbersome than in the two-dimensional case, since now the mesh consist of quadratic triangles. Triangles require two different types of refinement, namely regular and irregular [24] and a short description of the implementation can be found in the Appendix A. Furthermore, mesh refinement in three dimensions increases the size of the resulting system of equations considerably. For that reason, the GMRES iterative solver from the Sparskit library [25], with a modified preconditioner similar to [26] is employed.

\subsection{Solid mechanics}

To determine solid mechanical properties we perform uniaxial compression and shear simulations directly on the microstructures obtained from the foaming simulations. The matrix material is described by a linear elastic isotropic material model. Furthermore, the material is assumed to be under plane stress, i.e. the stress in the third direction is negligible, and material parameters Young's modulus $E_{s}=45 \mathrm{MPa}$ and Poisson ratio $\nu=0.5$. Quadratic elements are used to determine the material properties more accurately. The simulations are performed in MARC/MENTAT [27].

2.2.1 Boundary conditions A compression test is simulated to determine the Young's modulus $E$. A displacement of $\Delta z=0.01 \mathrm{~L}$ is applied in $P_{4}$ in $y$ direction. The reaction force, $F$, in this point is measured, which is used to calculated the stress

$$
\sigma=\frac{F}{A},
$$

where $A$ is the area of the computation domain. The Young's modulus can be expressed as:

$$
E=\frac{\sigma}{\epsilon} .
$$

where is $\epsilon$ is the applied strain. In Fig. 4a, the boundary conditions for the compression test are shown. The black arrows indicate the displacement is fixed. A shear test is performed to obtain the shear modulus, $G$. A displacement of $\Delta z=0.01 L$ in $x$ direction is applied in $P_{4}$. On the bubble boundaries stress free boundary conditions are imposed. The reaction force in this point is measured, which is transformed to the shear modulus as follows

$$
G=\frac{\tau}{\gamma},
$$

where $\tau=F / A$ is the shear stress and $\gamma$ the shear strain. In Fig. 4b, the applied boundary conditions for the shear test are shown. Again the black arrows indicate the displacement is fixed. 


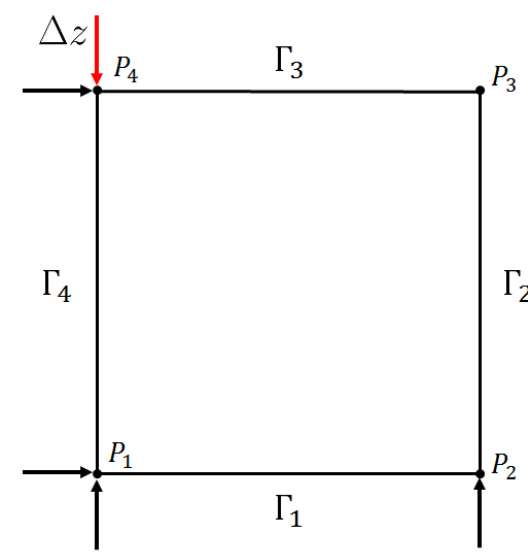

(a)

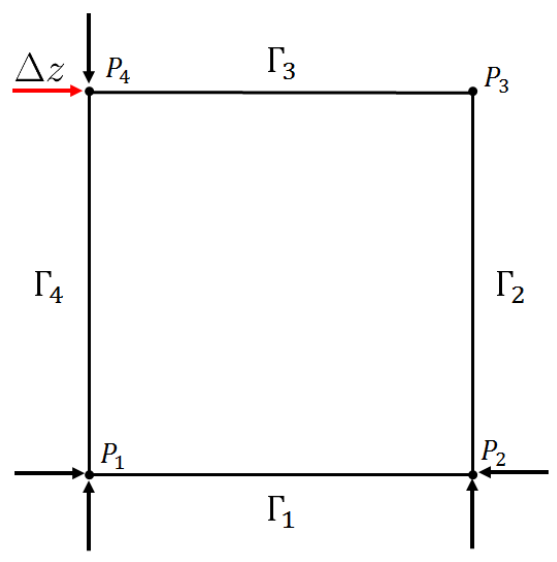

(b)

Fig. 4: Schematic representation of the applied boundary conditions for a) the compression test simulations and b) the shear test simulations. Black arrows indicate fixed displacement boundary condition, the red arrow indicates constant strain rate or constant shear rate boundary condition.

Periodic boundary conditions are used in the solid simulations because these determine the mechanical behaviour in foam structures more accurately [28]. The displacement of nodes on a curve are tied with the displacement of their corresponding nodes on opposite curves and to two corner nodes using SERVO-links, which can be summarized as [29],

$$
\begin{aligned}
& \left.\boldsymbol{d}\right|_{\Gamma_{4}}=\left.\boldsymbol{d}\right|_{\Gamma_{2}}-\left.\boldsymbol{d}\right|_{P_{2}}+\left.\boldsymbol{d}\right|_{P_{1}}, \\
& \left.\boldsymbol{d}\right|_{\Gamma_{3}}=\left.\boldsymbol{d}\right|_{\Gamma_{1}}-\left.\boldsymbol{d}\right|_{P_{1}}+\left.\boldsymbol{d}\right|_{P_{4}}
\end{aligned}
$$

Furthermore, the traction on opposite boundaries should be equal with opposite signs

$$
\begin{aligned}
& \left.\boldsymbol{t}\right|_{\Gamma_{2}}=-\left.\boldsymbol{t}\right|_{\Gamma_{4}}, \\
& \left.\boldsymbol{t}\right|_{\Gamma_{1}}=-\left.\boldsymbol{t}\right|_{\Gamma_{3}} .
\end{aligned}
$$

Since the air entrapped in the cells is a gas, the contribution of the pressure build-up to the stiffness can be neglected [5].

2.2.2 Extension of solid mechanics model in 3D The equivalent schematic representation in three dimensions can be seen in Fig. 5. An uniaxial compression test is performed to determine the Young's modulus, where a displacement $\Delta z=0.01 L$ is applied in negative $y$ direction in $P_{5}$. In the shear test, a displacement $\Delta z=0.01 L$ in $x$ direction is applied in $P_{5}$, to measure the shear modulus. In addition, the periodicity constraint is defined in all 


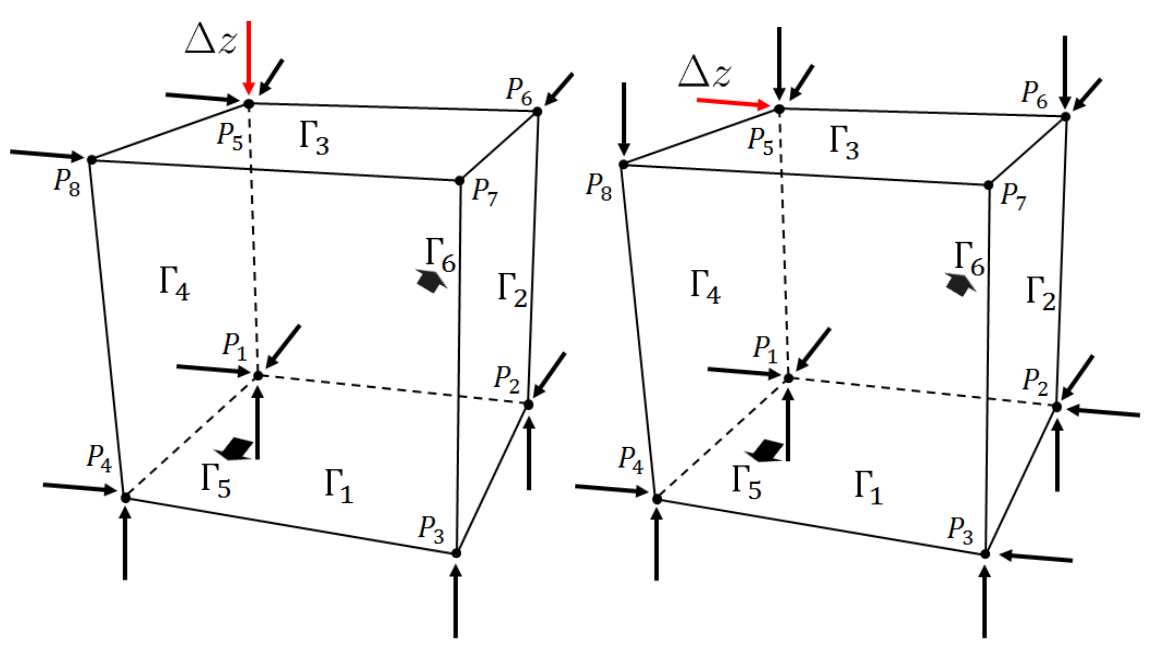

(a)

(b)

Fig. 5: Schematic representation of the applied boundary conditions for a) the compression simulation and b) the shear test simulations. The black arrows indicate fixed displacement boundary condition, the red arrow indicates constant strain or shear rate boundary condition.

three directions

$$
\begin{aligned}
& \left.\boldsymbol{d}\right|_{\Gamma_{4}}=\left.\boldsymbol{d}\right|_{\Gamma_{2}}-\left.\boldsymbol{d}\right|_{P_{2}}+\left.\boldsymbol{d}\right|_{P_{1}} \\
& \left.\boldsymbol{d}\right|_{\Gamma_{3}}=\left.\boldsymbol{d}\right|_{\Gamma_{1}}-\left.\boldsymbol{d}\right|_{P_{1}}+\left.\boldsymbol{d}\right|_{P_{5}} \\
& \left.\boldsymbol{d}\right|_{\Gamma_{5}}=\left.\boldsymbol{d}\right|_{\Gamma_{6}}-\left.\boldsymbol{d}\right|_{P_{1}}+\left.\boldsymbol{d}\right|_{P_{4}},
\end{aligned}
$$

and the traction on opposite boundaries in $z$-direction should be equal with opposite sign as well,

$$
\left.\boldsymbol{t}\right|_{\Gamma_{5}}=-\left.\boldsymbol{t}\right|_{\Gamma_{6}}
$$

\section{Results}

In the following section results for the bubble growth and the solid mechanical simulations will be presented both in two and three dimensions. The bubble growth simulations are first used to obtain the microstructures. These microstructures are then used directly in the solid mechanical simulations to obtain material parameters such as the Young's modulus and the shear modulus of the solidified foam. 


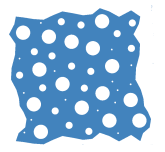

(a)

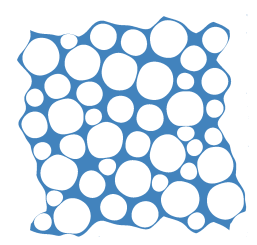

(b)

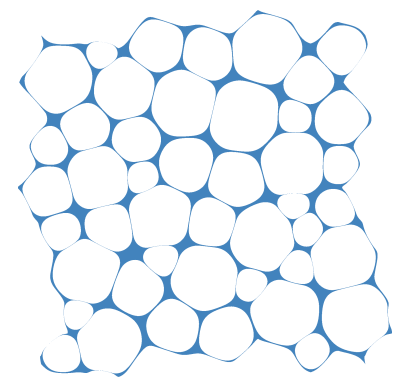

(c)

Fig. 6: Fluid simulation of a random distribution of bubbles with radius $R$, $R / 2$ and $R / 8$, at different times. a) Shows the initial microstructure with volume fraction $f=0.2, \mathrm{~b})$ shows the microstructure at $t=3.53$ with $f=0.69$, and c) shows the final microstructure at $t=5.3$ with $f=0.930$.

\subsection{Bubble growth in $2 D$}

In Fig. 6, the evolution of the microstructure is shown at different times, where three different bubble sizes with radius $R, R / 2$ and $R / 8$, are used in order to represent a more realistic structure. In the initial structure, bubbles are randomly distributed in the RVE. A convection-diffusion problem is solved for nodes on $\Gamma$ to create an initial smooth curved boundary that does not intersect with the bubbles [30]. Fig. 7, shows three microstructures which are generated using an $\alpha_{\mathrm{b}}=1.01,1.1,2.0$ for Fig. 7a-c respectively. If $\alpha_{\mathrm{b}}$ is small, the fluid in the thin layer has enough time to flow. Therefore the growth of the bubbles is not disturbed by the fluid and the shape of the bubbles remains circular. On the contrary, using a large $\alpha_{\mathrm{b}}$, the fluid does not have time to flow and this will effect the shape of the bubbles, as shown in Fig. 7c. In the simulations a growth parameter of $\alpha_{\mathrm{b}}=1.1$ is used.

3.1.1 Convergence tests In order to determine the accuracy of the simulation, it is important to perform convergence tests. First a spatial convergence test is conducted. Fluid simulations with the same initial microstructure are foamed until volume fraction $f=0.75$, with a time step of $\Delta t=5 \times 10^{-3}$. Finer meshes with more elements are obtained by increasing the refinement criteria. Refinement criteria as defined in Eq. (23) of $R_{\text {crit }}=0,1,2,3,6$ are considered and the corresponding number of nodes of the final structure can be found in Table 1 . Here, the case of $R_{\text {crit }}=0$ means no refinement. The relative error $e_{\mathrm{r}}$ of the size is determined at the end of the simulation and is given by

$$
e_{\mathrm{r}}=\left\|\frac{l_{s}-l_{s}^{*}}{l_{s}^{*}}\right\| \text {, }
$$




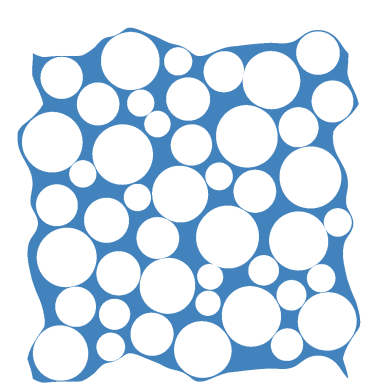

(a)

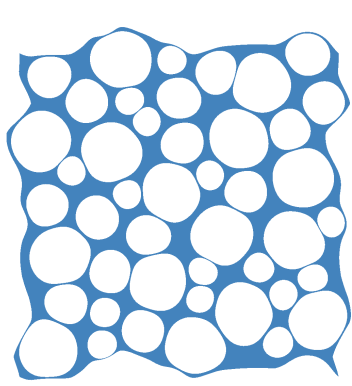

(b)

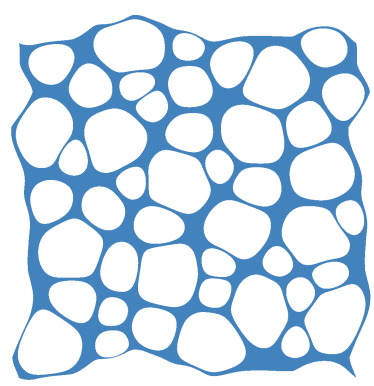

(c)

Fig. 7: Microstructures generated with growth parameter a) $\alpha_{\mathrm{b}}=1.01, \mathrm{~b}$ ) $\alpha_{\mathrm{b}}=1.1$ and c) $\alpha_{\mathrm{b}}=2.0$ at volume fraction of $f=0.75$.

Table 1: Number of nodes $N_{\text {nodes }}$ for meshes with different refinement criteria of $R_{\text {crit }}$, where $R_{\text {crit }}=0$ means no refinement.

\begin{tabular}{c|ccccc}
$R_{\text {crit }}$ & 0 & 1 & 2 & 3 & 6 \\
\hline$N_{\text {nodes }}$ & 3422 & 6162 & 17929 & 37442 & 132715
\end{tabular}

where $l_{s}$ is defined as the distance between points $P_{2}$ and $P_{1}$ for different meshes and $l_{s}^{*}$ is the reference solution of a mesh with refinement criteria of $R_{\text {crit }}=6$. In Fig. 8 the spatial convergence plot is shown, where a slope of three is visible, which is expected from the second-order elements in the microstructures.

Temporal convergence is studied on the same foaming simulations using refinement criteria of $R_{\text {crit }}=2$. The time step is varied between $\Delta t=$ $1 \times 10^{-1}$ and $\Delta t=5 \times 10^{-4}$. Fig. 9 shows the $e_{\mathrm{r}}$ as a function of the time step. The reference solution, $l_{s}^{*}$, is computed with a time step of $\Delta t=$ $5 \times 10^{-4}$. The slope in the graph is between one and two, which is better than expected, since the position of the nodes on the boundary box are updated using a first-order time integration.

We continue with determining the minimum number of elements that are required in the thin layer between growing bubbles to accurately describe the fluid flow dynamics. Fig. 10 shows a contour plot of the magnitude of the viscous stress tensor in the thin layer with refinement criteria 1, 2, and 3 showing at least 2, 4, or 6 elements between bubble interfaces respectively. The magnitude of the viscous stress tensor is defined as $2 \mu \sqrt{\boldsymbol{D}: \boldsymbol{D}}$. A refinement criteria of $R_{\text {crit }}=2$ is sufficient to describe the details of the flow dynamics between growing bubbles. In order to obtain accurate microstructures for the solid part of this article, the relative error in the fluid simulations is chosen to be below $0.05 \%$. Therefore in the fluid simulations, a refinement criteria of $R_{\text {crit }}=2$ and a time step of $\Delta t=5 \times 10^{-3}$ is used. 


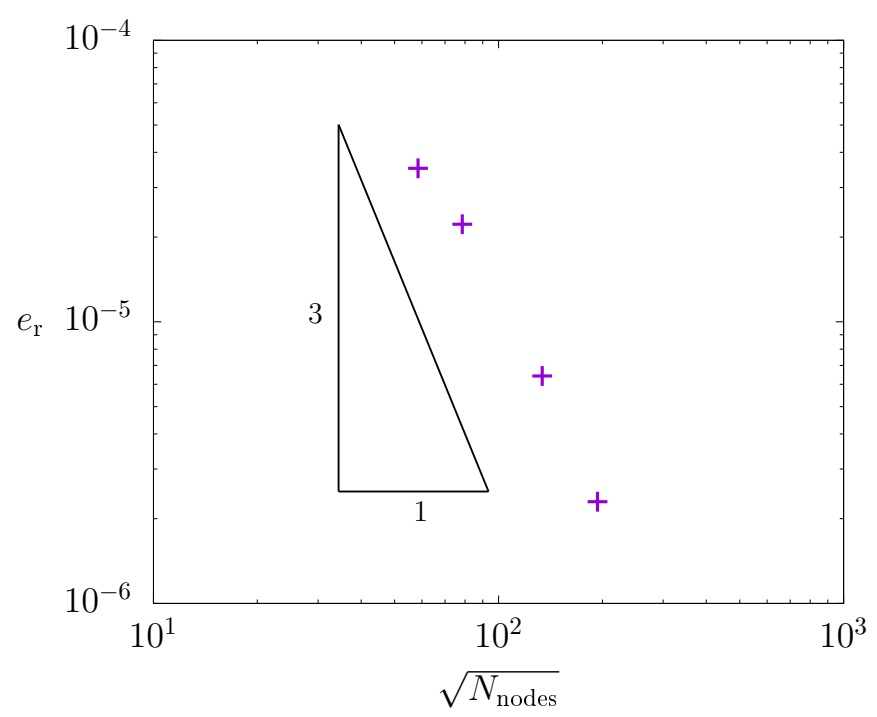

Fig. 8: Spatial convergence tests of $l_{s}$.

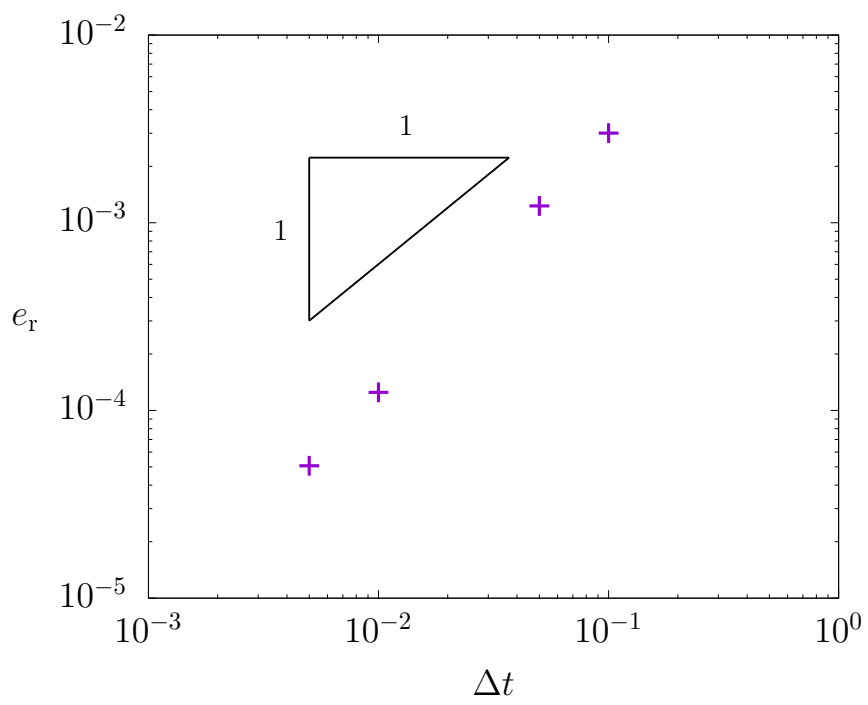

Fig. 9: Temporal convergence tests of $l_{s}$. 


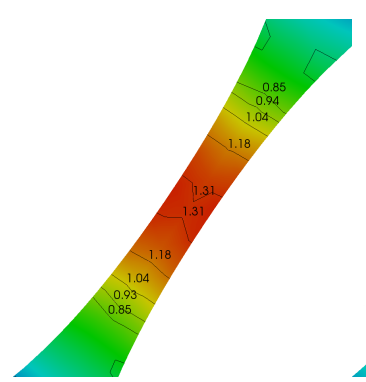

(a)

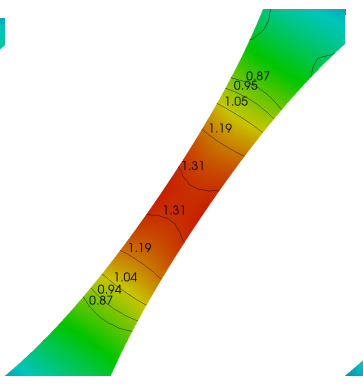

(b)

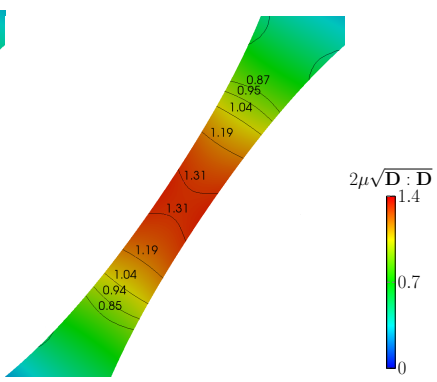

(c)

Fig. 10: Magnitude of the viscous stress tensor for different refinement criteria. In a) $R_{\text {crit }}=1$ giving 2 elements, b) $R_{\text {crit }}=2$ giving 4 elements, c) $R_{\text {crit }}=3$ giving 6 elements in the cross section between bubble interfaces.

3.1.2 Foaming microstructures in $2 D$ In Fig. 6a, the initial microstructure with bubbles randomly distributed over the periodic domain is depicted. The minimum distance between initially placed bubbles is equal to the maximum bubble radius of the given size distribution. During the foaming process the domain remains periodic. Fig. 6c shows the obtained microstructure after the simulation has reached a volume fraction of $f=0.930$. In Fig. 11, the volume fraction of bubbles in the bulk $f$ as a function of time is shown. When bubbles are perfect circles, the volume fraction increases linearly, as expected from the applied boundary conditions. The Laplace pressure from surface tension is estimated for a circular bubble, which will introduce an error when the bubbles are not spherical, resulting in a decrease in the slope.

Fig. 6c also shows that the fluid layers between bubbles can become extremely thin. In Fig. 12, elements are shown in the microstructure. A fluid plateau and a thin wall are magnified, to show that the elements are not distorted. To give an idea about the length scales, element lengths are shown in the magnified figures. The ratio of coarse element length to fine element length in the same microstructure is 94 , while the ratio of coarsest initial element length to finest element length is equal to 270 .

\subsection{Bubble growth in $3 D$}

The foaming microstructure at different times is depicted in Fig. 13. In the initial structure, bubbles are randomly distributed in the RVE. The nodes on the surface mesh are moved outside of the bubbles in an iterative process, to prevent intersection with the bubbles, which creates the initial random box similar to [18].

3.2.1 Convergence tests Convergence tests are performed to determine the accuracy of the simulation. First a spatial convergence test is conducted. 


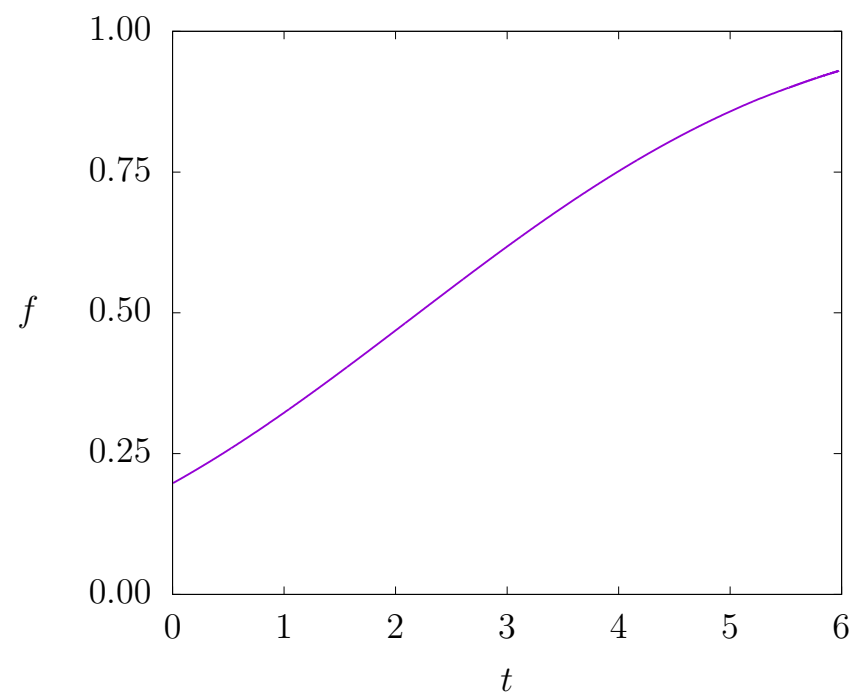

Fig. 11: Bubble volume fraction $f$ as a function of time for a microstructure with 45 bubbles.

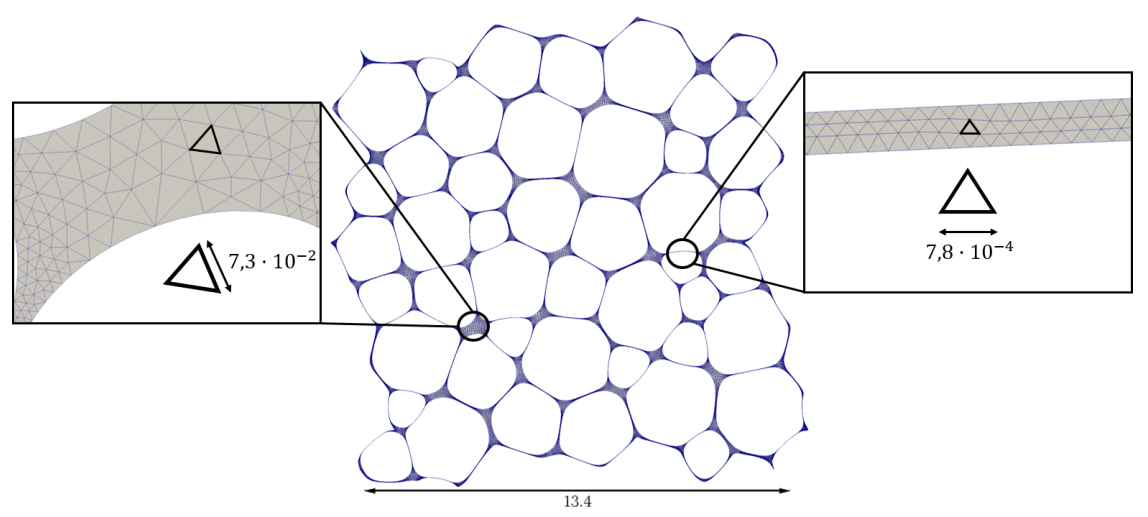

Fig. 12: In the left, a fluid plateau is magnified, showing coarse elements with length $7.3 \times 10^{-3}$. On the right, a thin fluid layer is magnified, showing fine elements with length $7.8 \times 10^{-4}$.

Microstructures with one bubble in the computational domain are simulated until a volume fraction $f=0.55$ is reached, with a time step of $\Delta t=5 \times 10^{-3}$. Finer meshes are obtained by increasing the refinement criteria. Refinement criteria of $R_{\text {crit }}=0.7,1.5,2,2.5$ and 3 are considered with corresponding number of nodes in the final structure as shown in Table 2. The relative error $e_{\mathrm{r}}$ of the size is defined in Eq. (37). The reference solution is computed on a mesh with refinement criteria $R_{\text {crit }}=3$. In Fig. 14, the 


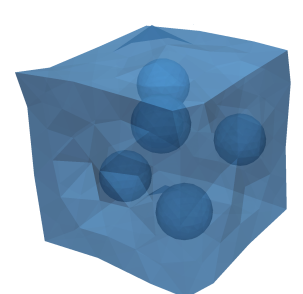

(a)

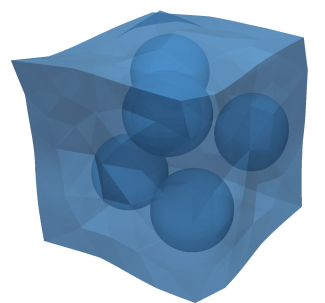

(b)

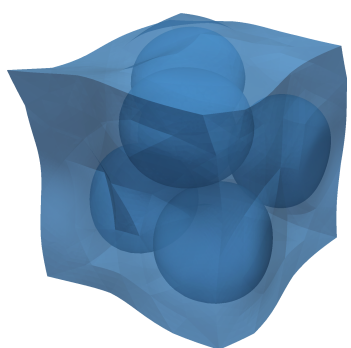

(c)

Fig. 13: Fluid simulation at different times. a) Shows the initial microstructure with volume fraction $f=0.08, \mathrm{~b}$ ) shows the microstructure at $t=3.53$ with $f=0.20$, and $\mathrm{c}$ ) shows the final microstructure at $t=5.3$ with $f=0.42$.

Table 2: Number of nodes $N_{\text {nodes }}$ for meshes with different refinement criteria of $R_{\text {crit }}$.

\begin{tabular}{c|ccccc}
$R_{\text {crit }}$ & 0.7 & 1.5 & 2 & 2.5 & 3 \\
\hline$N_{\text {nodes }}$ & 16625 & 63937 & 125648 & 219802 & 334578
\end{tabular}

space convergence plot is shown. A third-order convergence can be seen, as expected from the quadratic elements.

Temporal convergence is studied on the same foaming simulation using a refinement criteria $R_{\text {crit }}=1.5$. The time step is varied between $\Delta t=$ $5 \times 10^{-2}$ and $\Delta t=1 \times 10^{-3}$. Fig. 15 shows the relative error of the size as a function of the time step. The reference solution, $l_{s}^{*}$, is computed with a time step of $\Delta t=5 \times 10^{-4}$. The size convergence with a slope slightly higher as one, which is better than expected because the position of the nodes on the boundary box are updated using a first-order time integration scheme.

To obtain accurate microstructures which will be used as input for the solid mechanics simulations, the relative error $e_{\mathrm{r}}$ is chosen to be below $0.05 \%$. Therefore it is sufficient to use a refinement criteria $R_{\text {crit }}=1.5$ and a time step of $\Delta t=5 \times 10^{-3}$.

3.2.2 Foaming microstructures in 3D Fig. 13a shows the initial periodic microstructure. The minimum distance between initially placed bubbles is equal to the maximum bubble radius. During the fluid simulation, the domain always remains periodic. Fig. 13c shows the microstructure after the simulation has reached a volume fraction $f=0.42$. In Fig. 16, the mesh of the outer boundary box is shown at different times. Regular and irregular refined elements are clearly visible.

In Fig. 17 a cross section of the microstructure is shown. The thin layer of fluid is magnified to show the elements are not distorted. To give an idea 


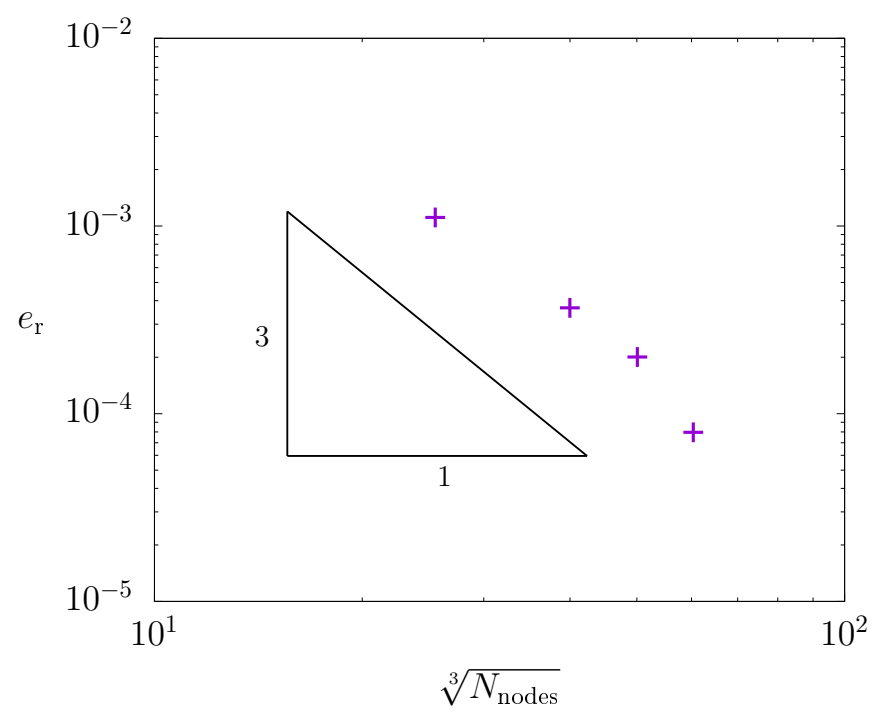

Fig. 14: Spatial convergence tests of $l_{s}$.

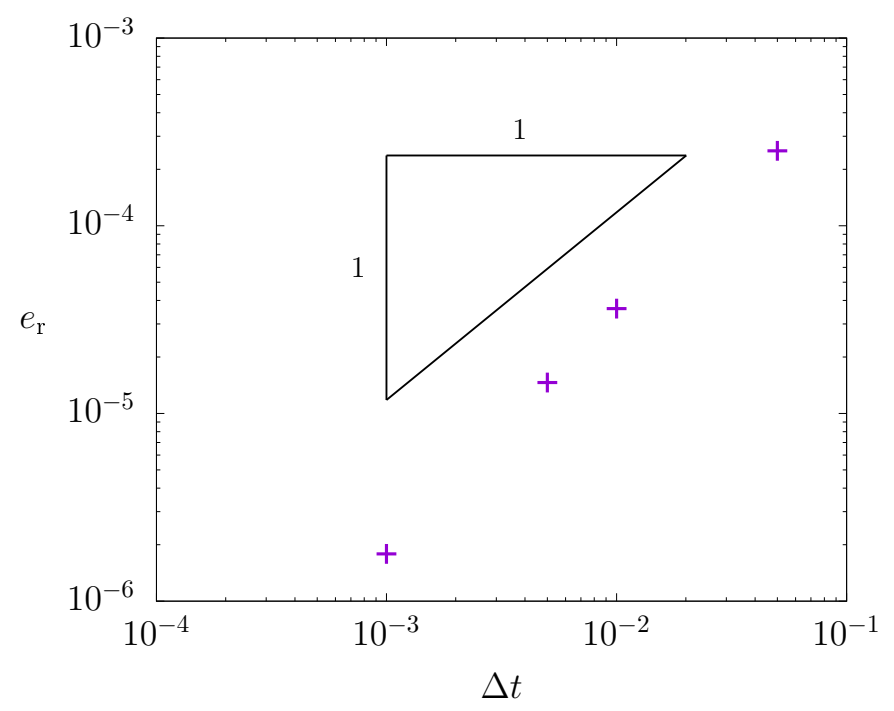

Fig. 15: Temporal convergence tests of $l_{s}$. 


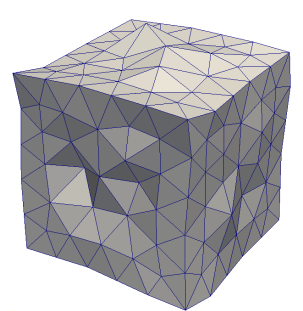

(a)

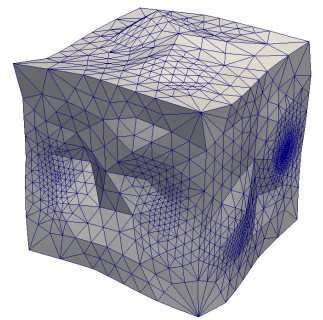

(b)

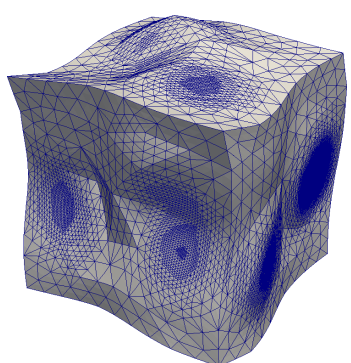

(c)

Fig. 16: Mesh refinement during the fluid simulation of the outer boundary box at different times. a) shows the initial microstructure with volume fraction $f=0.08$, and $\mathrm{c}$ ) shows the final microstructure with $f=0.42$.
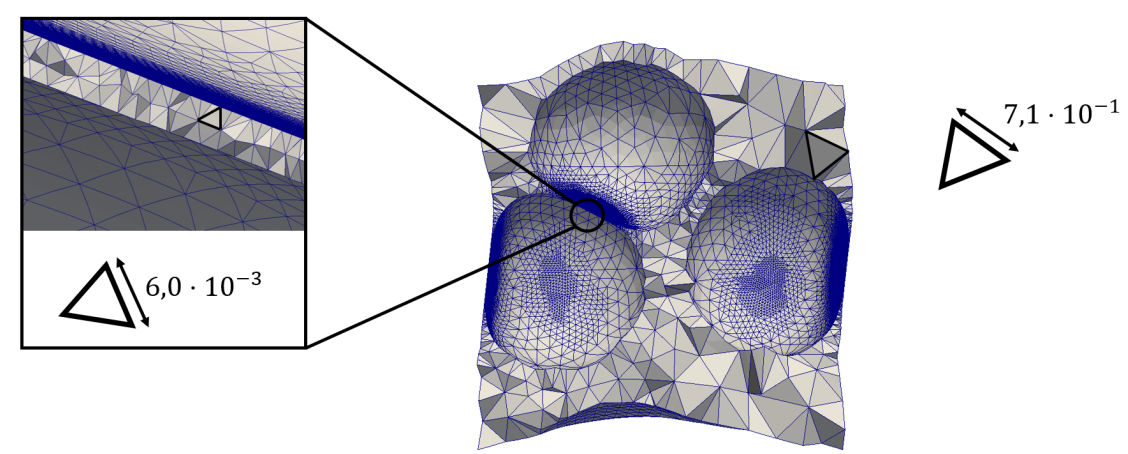

Fig. 17: Cross section of the microstructure. On the left a thin fluid layer is magnified, showing fine elements with an element length of $6.0 \times 10^{-3}$. Coarse elements with element length $7.1 \times 10^{-1}$ are shown on the right.

about the considered length scales, the element length of two elements are shown. The ratio of coarsest element length to finest element length is 120 .

\subsection{Solid foam mechanics in $2 D$}

The microstructures generated using the bubble growth simulations are assumed to have been completely solidified. The geometries are saved and can be used directly in solid mechanics simulations to obtain the material properties of these microstructures.

3.3.1 Convergence tests The spatial convergence of uniaxial compression and shear simulations is studied on a microstructure with ten bubbles and 
Table 3: Number of nodes $N_{\text {nodes }}$ for meshes with different refinement criteria of $R_{\text {crit }}$.

\begin{tabular}{c|ccccc}
$R_{\text {crit }}$ & 1 & 2 & 3 & 4 & 6 \\
\hline$N_{\text {nodes }}$ & 9136 & 14774 & 26396 & 43641 & 91993
\end{tabular}

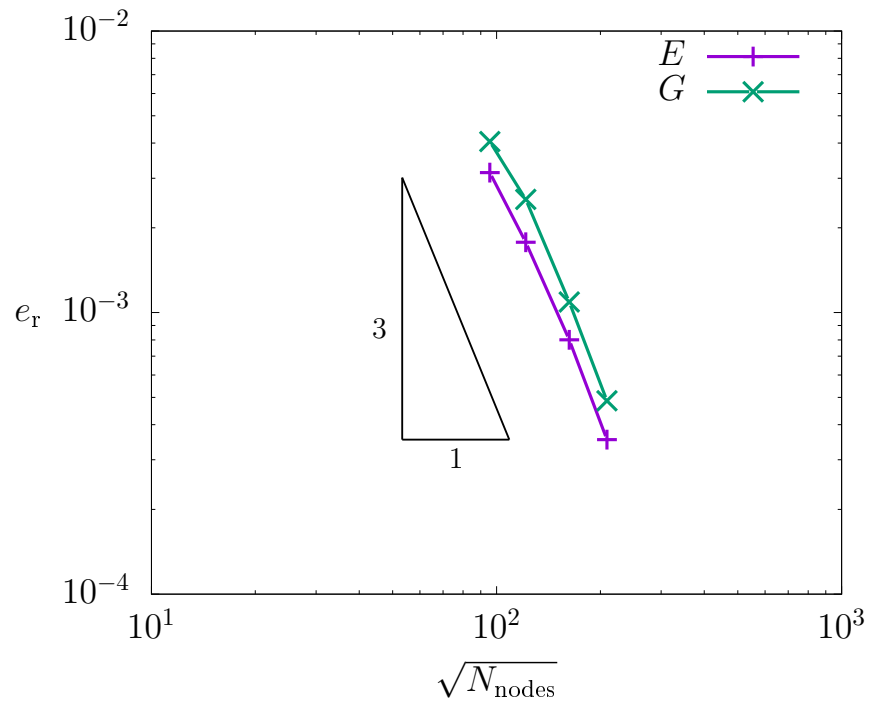

Fig. 18: Spatial convergence tests of the Young's modulus and the shear modulus.

a volume fraction of $45 \%$. Finer meshes are generated by changing the refinement criteria in the fluid simulations. Refinement criteria of $R_{\text {crit }}=$ $1,2,3,4,6$ are considered with corresponding number of nodes as seen in Table 3. Uniaxial compression and shear simulations are performed by applying a displacement of $\Delta z=0.1$. The relative error of the Young's modulus and the shear modulus are evaluated in a similar manner as in Eq. (37). In Fig. 18, the space convergence plot is shown. As expected from the use of quadratic elements, the shear modulus and the Young's modulus have third-order convergence. To remain below an error of 0.5 , the microstructures considered in the $2 \mathrm{D}$ solid simulations are generated with a refinement criteria $R_{\text {crit }}=2$.

3.3.2 Minimum number of bubbles in a RVE In order to call a microstructure a RVE, the mechanical properties should not be a function of the bubble distribution. This condition holds for isotropic materials. By increasing the number of bubbles, the standard deviation of the mechanical properties decreases. For a given number of bubbles, ten microstructures are generated with a volume fraction $f=0.90$ in order to obtain the material parameters 


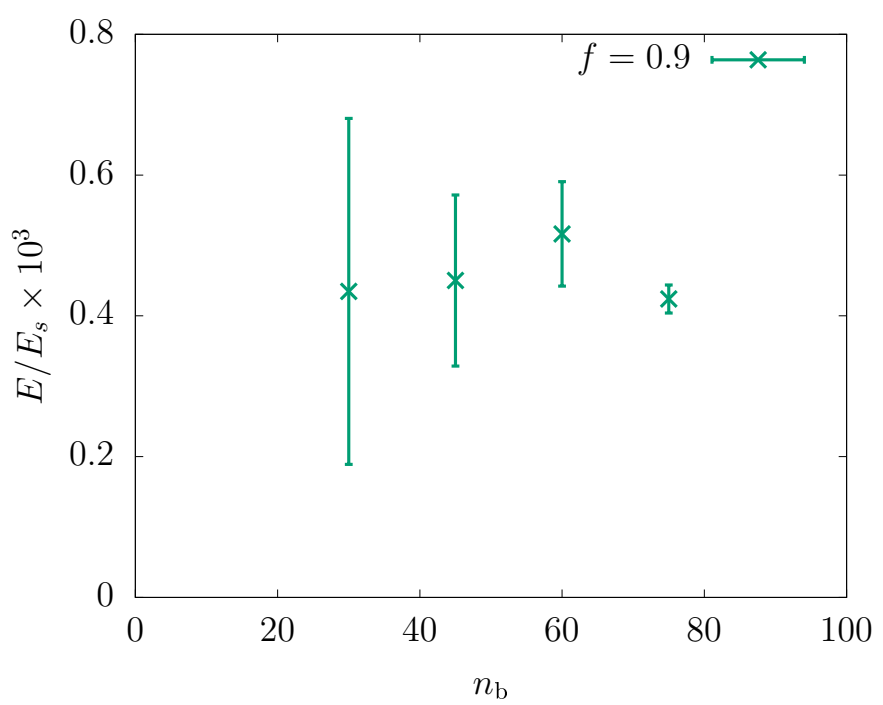

Fig. 19: The normalized Young's modulus for different microstructures as a function of the number of bubbles.

by averaging. To make the structures more realistic, initially, three different bubble sizes are considered: bubbles with radius $R, R / 2$ and $R / 8$. Every size consists of the same number of bubbles. Bubbles are placed, such that the distance between the center of two bubbles is at least $3 R$.

In Fig. 19-20, the Young's modulus and the shear modulus with their standard deviation are shown as a function of the number of bubbles respectively. The $y$-axis is normalized with the Young's modulus of the solid material. Fig. 19 shows that 30, 45 and 60 bubbles are not enough to determine the Young's modulus since the standard deviation remains large. The Young's modulus for microstructures with 75 bubbles in the bulk is however sufficiently accurate. Similarly, for the shear tests simulations, it can be seen, in Fig. 20, that 75 bubbles in the microstructures are required to determine the shear modulus sufficiently accurate.

\subsection{Solid foam mechanics in $3 D$}

Similar to the 2D case, after running simulations using the 3D bubble growth model, to obtain the desired microstructures, we assume that our foam solidifies and the geometries are used directly in solid mechanics simulations.

3.4.1 Convergence tests The space convergence of uniaxial compression and shear simulations is studied on microstructures with 15 bubbles having a volume fraction $f=0.13$. Finer meshes are obtained by changing 


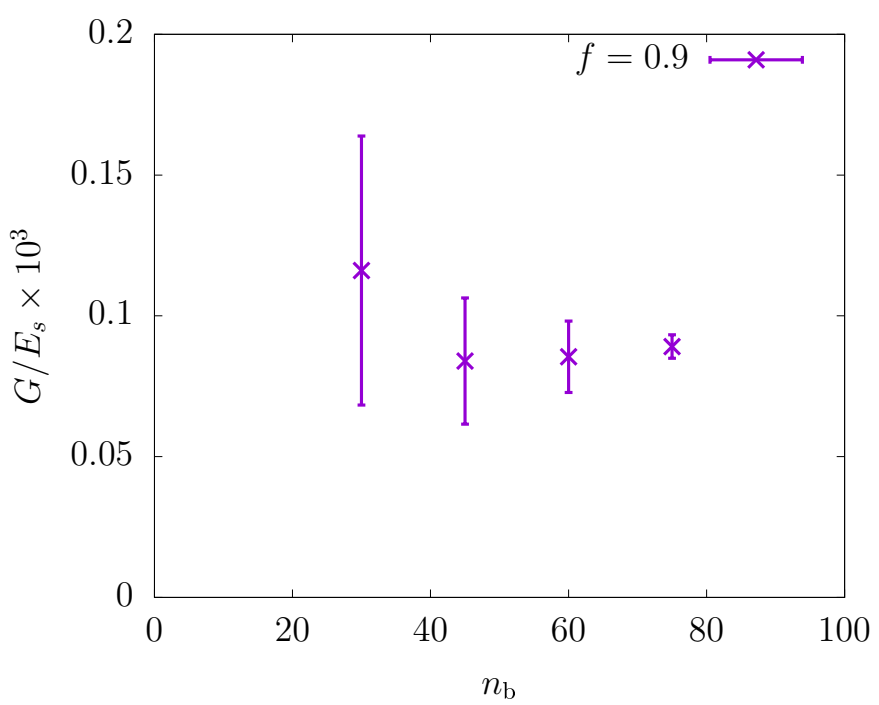

Fig. 20: The normalized shear modulus for different microstructures as a function of the number of bubbles.

Table 4: Number of nodes $N_{\text {nodes }}$ for meshes with different refinement criteria of $R_{\text {crit }}$.

\begin{tabular}{c|ccccc}
$R_{\text {crit }}$ & 2 & 3 & 4 & 4.5 & 5 \\
\hline$N_{\text {nodes }}$ & 59909 & 92311 & 160677 & 232308 & 386913
\end{tabular}

the refinement criteria, $R_{\text {crit }}$, in the fluid simulations. Microstructures are generate with $R_{\text {crit }}$ and the corresponding number of nodes in the domain can be found in Table 4. Uniaxial compression and shear simulations performed by applying a displacement of $\Delta z=0.01 \mathrm{~L}$. The reference solutions are computed on a mesh with $R_{\text {crit }}=5$. Fig. 21 shows the resulting space convergence plot. As expected, a slope of three is visible since quadratic elements are used. To remain below a relative error of $1 \%$, it is sufficient to use a refinement criteria of 2 .

3.4.2 Finite domain-size effect Due to the small number of bubbles used in the $3 \mathrm{D}$ simulations it is expected to see some localization effects. To study this effect multiple microstructures would need to be generated using the $3 \mathrm{D}$ bubble growth model, which can be very time consuming. To be able to check the effect of a chosen microstructure on the obtained mechanical properties, one can take advantage of the anisotropy of the given geometry. Thus, by using the same microstructure shown in Fig. 13, but rotated by 90 degree around the $x$ - and $z$-axis we are able to create two new microstructures. The average material properties are determined: $E / E_{s} \times 1000=476.6 \pm 11.45$, 


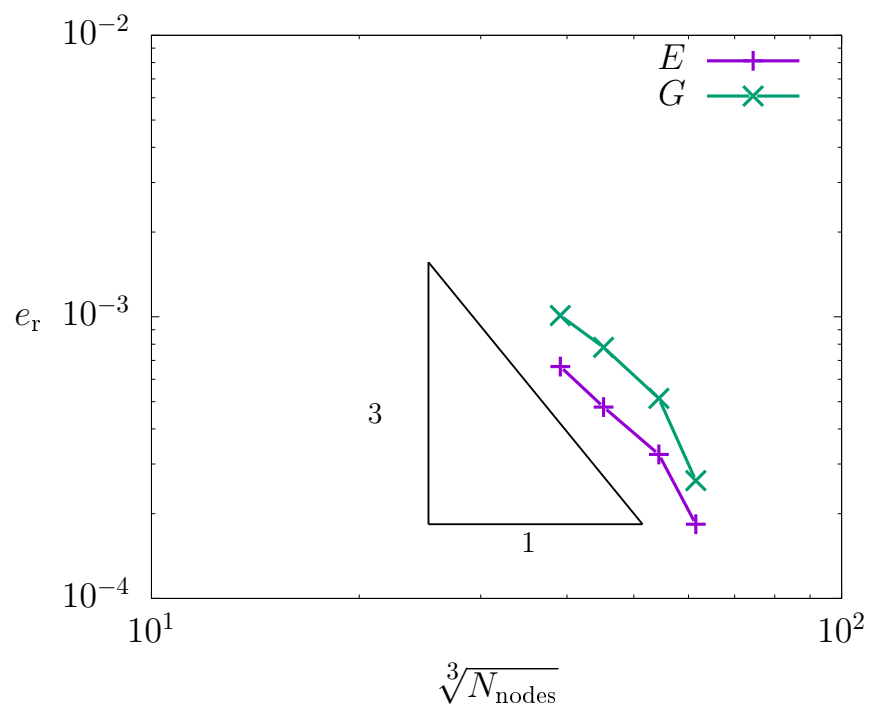

Fig. 21: Spatial convergence tests of the Young's modulus and the shear modulus in 3D.

and $G / E_{s} \times 1000=136.3 \pm 8.00$. The observed standard deviations are minor, for the small number of bubbles used, but this is expected due to the low volume fractions reached. Nevertheless, in order to properly study the required minimum number of bubbles more simulations with an increased number of bubbles would be needed.

\section{Conclusion}

We have presented direct numerical simulations both in 2D and 3D of foaming microstructures using representative volume elements. Such simulations allow us to predict the final geometry of foaming systems under the influence of processing conditions, i.e., flow. Initially bubbles were randomly distributed in an incompressible Newtonian fluid, where inertia forces are neglected. To ensure growth of the bubbles, a hydrostatic pressure was applied on the bubble interface larger than the estimated Laplace pressure resulting from surface tension. Mesh refinement in thin fluid layers is implemented to accurately describe the hydrodynamics between bubble interfaces. The mass balance, momentum balance and interface tracking equations are solved coupled to make the time step independent of the characteristic mesh size time limit. Spatial and temporal convergence tests were performed to determine the accuracy of the simulations. Microstructures were generated having a volume fraction of bubbles in the bulk of over $90 \%$, in $2 \mathrm{D}$ and $45 \%$ in 3D. These microstructures were then used directly in solid mechanics simulations were uniaxial compression and shear simulations were performed to 
obtain the Young's and shear moduli. Spatial convergence tests on these mechanical properties were performed to determine the accuracy of the simulations. The minimum required number of bubbles in a $2 \mathrm{D}$ microstructure was investigated, in order to obtain results that are independent of the initial distribution and size of the computational domain. For the 3D simulations, obtaining multiple microstructures can be extremely time consuming. Thus, we took advantage of the anisotropy of the system by rotating the microstructures around the $x$ - and $z$-axis and compared the results to study the finite domain-size effect due to the small number of bubbles used. Nevertheless, to properly study localization effects in 3D, more bubbles with an increased volume fraction will be needed. This can become feasible, by parallelizing the code in order to substantially reduce the computational time.

\section{Acknowledgment}

The research leading to these results has received funding from the European Commission under the grant agreement number 604271 (Project acronym: MoDeNa; call identifier: FP7-NMP-2013-SMALL-7)

\section{References}

1. W. Y. Jang, A. M. Kraynik, and S. Kyriakides, "On the microstructure of open-cell foams and its effect on elastic properties," International Journal of Solids and Structures, vol. 45, no. 7-8, pp. 1845-1875, 2008.

2. W. Y. Jang and S. Kyriakides, "On the crushing of aluminum open-cell foams: Part II analysis," International Journal of Solids and Structures, vol. 46, no. 34, pp. 635-650, 2009.

3. W. Y. Jang, S. Kyriakides, and A. M. Kraynik, "On the compressive strength of open-cell metal foams with Kelvin and random cell structures," International Journal of Solids and Structures, vol. 47, no. 21, pp. 2872-2883, 2010.

4. S. Gaitanaros, S. Kyriakides, and A. M. Kraynik, "On the crushing response of random open-cell foams," International Journal of Solids and Structures, vol. 49, no. 19-20, pp. 2733-2743, 2012.

5. L. J. Gibson and M. F. Ashby, Cellular Solids. Cambridge: Cambridge University Press, 1997.

6. M. Ashby, "The properties of foams and lattices," Philosophical Transactions of the Royal Society A: Mathematical, Physical and Engineering Sciences, vol. 364, no. 1838, pp. 15-30, 2006.

7. L. J. Gibson, M. F. Ashby, G. N. Karam, U. Wegst, and H. R. Shercliff, "The mechanical properties of natural materials. II: Microstructures for mechanical efficiency," Proceedings of the Royal Society of London A, vol. 450, no. 1938, pp. 141-162, 1995.

8. M. Karimi and D. L. Marchisio, "A Baseline Model for the Simulation of Polyurethane Foams via the Population Balance Equation," Macromolecular Theory and Simulations, vol. 24, no. 4, pp. 291-300, 2015. 
9. P. Ferkl, M. Karimi, D. L. Marchisio, and J. Kosek, "Multi-scale modelling of expanding polyurethane foams: Coupling macro- and bubble-scales," Chemical Engineering Science, vol. 148, pp. 55-64, 2016.

10. A. Arefmanesh and S. G. Advani, "Diffusion-induced growth of a gas bubble in a viscoelastic fluid," Rheologica Acta, vol. 30, no. 3, pp. 274-283, 1991.

11. N. S. Ramesh, D. H. Rasmussen, and G. A. Campbell, "Numerical and experimental studies of bubble growth during the microcellular foaming process," Polymer Engineering \& Science, vol. 31, no. 23, pp. 1657-1664, 1991.

12. M. Amon and D. C. Denson, "A Study of the Dynamics of foam growth Analysis of the growth of closely spaced spherical bubbles," Polymer Engineering and Science, vol. 24, no. 13, pp. 1026-1034, 1984.

13. M. Amon and C. D. Denson, "A Study of the Dynamics of Foam Growth Simplified Analysis and Experimental Results for Bulk-Density in Structural Foam Molding," Polymer Engineering and Science, vol. 26, no. 3, pp. 255-267, 1986.

14. S. L. Everitt, O. G. Harlen, and H. J. Wilson, "Bubble growth in a twodimensional viscoelastic foam," Journal of Non-Newtonian Fluid Mechanics, vol. 137 , no. 1-3, pp. 46-59, 2006.

15. J. J. Feng and C. A. Bertelo, "Prediction of bubble growth and size distribution in polymer foaming based on a new heterogeneous nucleation model," Journal of Rheology, vol. 48, no. 2, pp. 439-462, 2004.

16. P. Yue, J. J. Feng, C. A. Bertelo, and H. H. Hu, "An arbitrary LagrangianEulerian method for simulating bubble growth in polymer foaming," Journal of Computational Physics, vol. 226, no. 2, pp. 2229-2249, 2007.

17. S. L. Everitt, O. G. Harlen, and H. J. Wilson, "Competition and interaction of polydisperse bubbles in polymer foams," Journal of Non-Newtonian Fluid Mechanics, vol. 137, no. 1-3, pp. 60-71, 2006.

18. C. Mitrias, N. O. Jaensson, M. A. Hulsen, and P. D. Anderson, "Direct numerical simulation of a bubble suspension in small amplitude oscillatory shear flow," Rheologica Acta, vol. 56, no. 6, pp. 555-565, 2017.

19. M. M. Villone, M. A. Hulsen, P. D. Anderson, and P. L. Maffettone, "Simulations of deformable systems in fluids under shear flow using an arbitrary Lagrangian Eulerian technique," Computers and Fluids, vol. 90, pp. 88-100, 2014.

20. J. U. Brackbill, D. B. Kothe, and C. Zemach, "A continuum method for modeling surface tension," Journal of Computational Physics, vol. 100, no. 2, pp. 335-354, 1992.

21. HSL, "A collection of Fortran codes for large scale scientific computation," 2013.

22. H. H. Hu, N. A. Patankar, and M. Y. Zhu, "Direct Numerical Simulations of Fluid-Solid Systems Using the Arbitrary Lagrangian-Eulerian Technique," Journal of Computational Physics, vol. 169, no. 2, pp. 427-462, 2001.

23. C. Geuzaine and J.-F. Remacle, "Gmsh: A 3-D finite element mesh generator with built-in pre- and post-processing facilities," International Journal for Numerical Methods in Engineering, no. 79, pp. 1309-1331, 2009.

24. H. Yserentant, "Two Multi-Level Methods for Nonuniformly Refined Grids," in Computer Algorithms for Solving Linear Algebraic Equations (E. Spedicato, ed.), (Berlin, Heidelberg), pp. 161-167, Springer Berlin Heidelberg, 1991.

25. Y. Saad, "SPARSKIT: A Basic Tool Kit for Sparse Matrix Computations," tech. rep., NASA Ames Research Center, 2001. 
26. N. O. Jaensson, C. Mitrias, M. A. Hulsen, and P. D. Anderson, "Shear-Induced Migration of Rigid Particles near an Interface between a Newtonian and a Viscoelastic Fluid," Langmuir, vol. 34, no. 4, pp. 1795-1806, 2018.

27. MARC/MENTAT, "http://www.mscsoftware.com/product/marc," 2014.

28. C. Chen, T. J. Lu, and N. A. Fleck, "Effect of imperfections on the yielding of two-dimensional foams," Journal of the Mechanics and Physics of Solids, vol. 47, no. 11, pp. 2235-2272, 1999.

29. S. Krop, H. E. Meijer, and L. C. Van Breemen, "Global and local largedeformation response of sub-micron, soft- and hard-particle filled polycarbonate," Journal of the Mechanics and Physics of Solids, vol. 87, pp. 51-64, 2016.

30. N. O. Jaensson, M. A. Hulsen, and P. D. Anderson, "Stokes-Cahn-Hilliard formulations and simulations of two-phase flows with suspended rigid particles," Computers and Fluids, vol. 111, pp. 1-17, 2015.

\section{A Refining in 3D}

Mesh refinement is needed to accurately describe the fluid dynamics in the thin layer between bubbles. However, the refinement of the mesh in three dimensions is more cumbersome than in the two-dimensional case, since now the mesh consist of quadratic triangles. Triangles require two different types of refinement, regular and irregular refinement [24]. Regular refinement splits one triangular surface element into four new ones and irregular refinement splits one triangular surface element into two new ones, see Fig. 22a. Elements generated during irregular refinement are called green elements. An element before refinement is called the parent element. During refinement, new smaller elements are created, which are called siblings. Regular refined elements will therefore have four siblings, while irregular refined elements will have only two. Every element has its own refinement level and by regular refinement the level is increased by one.

While refining a single element, unwanted hanging nodes would be generated on its sides, if the neighboring elements were not refined as well, see Fig. 22b. When an element is marked for regular refinement (M), its neighboring elements are marked to be irregularly refined $(G)$ in order to get rid of hanging nodes, see Fig. 23a. When a green element is marked for refinement (Fig. 23b), the irregular refinement is first removed and the parent element is regularly refined, as in Fig. 23c. The elements in the mesh are refined level by level, starting with elements with the lowest refinement level, to get rid of hanging node that are generated when two neighboring elements are refined and are of different refinement level, see Fig. 23d. In Fig. 23 some basic cases of mesh refinement previously mentioned, are shown. In every next figure, the marked elements in the previous figure are refined. 


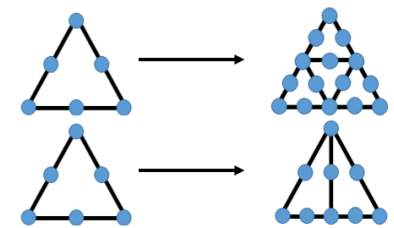

(a)

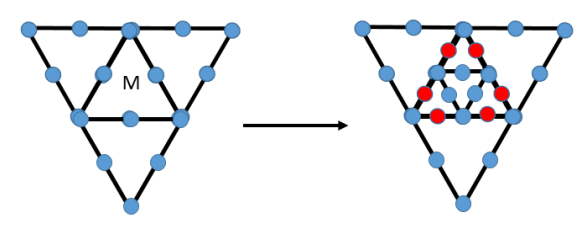

(b)

Fig. 22: a) Regular refinement (top) and irregular refinement (bottom) of an element. The blue circles are the location of the nodes. b) Regular refinement of marked element (M). The neighboring elements are not refined and hanging nodes are created (red nodes). To avoid hanging nodes, the neighboring elements are irregularly refined.

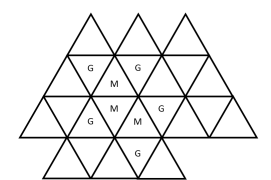

(a)

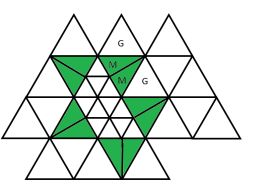

(b)

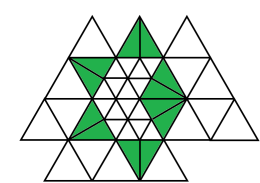

(c)

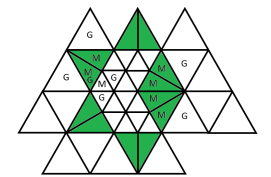

(d)

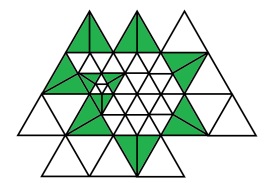

(e)

Fig. 23: a) Three elements are marked for regular refinement(M), as a result 5 neighboring elements are marked for irregular refinement $(G)$, to withhold the creation of hanging nodes. b) Green elements marked for refinement. c) Regular refinement of green elements. d) On the left, two neighboring elements of different refinement level are marked, which results in an element that is marked for both regular refinement and for irregular refinement. This cannot be done, however to solve this, refinement is done level by level. The marked elements on the right show that no double green elements can be created. e) The final obtained structure. 